\title{
Circular Coil for EV Wireless Charging Design and Optimization Considering Ferrite Saturation
}

\author{
F. Corti, F. Grasso, L. Paolucci \\ Dept. of Information Engineering \\ University of Florence, Italy \\ \{fabio.corti, francesco.grasso, libero.paolucci\}@unifi.it
}

\author{
L. Pugi, L. Luchetti \\ Dept. of Industrial Engineering \\ University of Florence, Italy \\ luca.pugi@unifi.it
}

\begin{abstract}
In this paper a circular coil for electric vehicle (EV) static wireless charging is considered. In particular, the coil geometry has been optimized in order to maximize coupling coefficient, reduce losses and thus maximizing transmission efficiency. The procedure that has been used to optimize the ferrite thicknesses to prevent the material saturation is shown and the losses introduced from the aluminum shielding are estimated. In addition, the attention was focused on magnetic fields limits in surrounding environment and on the system cost. The results obtained from finite element method (FEM) simulation have been experimentally validated.
\end{abstract}

Keywords: Wireless Power Transfer, Ferrite, Coils, Magnetics, Core saturation

\section{INTRODUCTION}

The electric vehicle market has been growing considerably in recent years. The economic incentives policies that various countries are adopting, batteries cost reduction, and the improvement in power electronics have boosted its spread. Thanks to these factors electric vehicle cost has gradually been reduced by making it accessible to a large number of people. Therefore, in a scenario where internal combustion vehicles will be probably replaced by electric vehicles, the wireless charging (WC) seems to be an interesting strategy able to facilitate the EV changing process. The interest on this topic from industries and academic institution is constantly increasing. Several studies have been carried out to understand the conditions under which the WC becomes more convenient than wired charging. For example, in [1] the application of WC is studied for a garbage collection service. The results highlight the possibility of equipping the vehicle with a smaller battery pack with consequent reduction of weight, space and costs.

Besides the automotive sector, in literature it is possible to find studies concerning the use of wireless transmission systems also for different applications. In [2], wireless charging for railway application is studied while in [3] a wireless charging system for underwater robotic is shown.

Regardless from the applications, the coil geometry plays a key role to achieve high transmission efficiency. In [4], an overview on the coil geometries for $\mathrm{EV}$ wireless charging is presented. As shown in the paper, the first and most common coil geometry for WC is the circular one. In order to facilitate a low reluctant flux path and to reduce the flux leakage, ferrite bars are usually used. Also, an aluminum plate is placed at the back of the whole coil to hold up the flux distribution. This topology takes the advantage that the structure is easily buildable with the symmetric flux distribution around the center. With the same occupied surface, the least amount of copper is used. For this reason, this coil is the cheapest. On the other hand, the mutual inductance is very sensitive to misalignment. Thus, the ferrite bars positioning and the geometry optimization plays a key role to achieve high coupling. Many studies have been carried out to identify different geometries able to guarantee good couplings even in conditions of misalignments . For example, the double D quadrature polarized coil (DDQP) was designing to generate the both parallel and perpendicular magnetic field. Since the DDQP topology can generate the polarized and non-polarized field by regulating the coil current, the system flexibility is higher than other topologies. But the drawback is the increased number of coils, which leads to the increased cost. Comparing to the DDQP topology, another high flexibility topology called bipolar pad (BPP) was introduced to generating the parallel and perpendicular filed, where the two coils are partially overlapped. In the meantime, the system complexity and cost are reduced by eliminating one coil.

Since the cost and weight of a coil depends on the quantity of copper, ferrite and aluminum that is used, in this paper the attention is focused on the circular coil representing the cheapest solution. In addition, even if it is not the coil that guarantees the best performance in case of misalignment, in a future where autonomous vehicle operation will be widely exploited, precise positioning of the vehicle with respect to the coil will be easily achieved. Starting from [4]-[8], a study of the effect of ferrite saturation is carried out performing FEM simulations. Then, the results can be used to produce a piecewise linearized models that should be exploited for the electric simulation of the system using standard lumped models.

\section{PlanAR Circular COIL}

\section{A. System Requirements}

According to the SAE J2954 standard, the system was designed to work at an operating frequency of $f=85 \mathrm{kHz}$. It is assumed that the primary coil is geometrically equal to the secondary one. Since one of the two has to be mounted on the vehicle, its dimension was limited to a maximum diameter of 
$D_{\max }=50 \mathrm{~cm}$. It is assumed that the current through the coils is sinusoidal with a maximum amplitude of $I_{\max }=100 \mathrm{~A}$. To avoid overheating due to power losses caused by skin effect and an excessively limited section, a Litz wire made up from of 2875 strand with a $38 \mathrm{AWG}$ diameter was selected. The coil distance is fixed to $d=15 \mathrm{~cm}$, and misalignment was not taken into account.

\section{B. FEM Analysis Without Ferrite}

The first step of the analysis was the coil geometry definition without considering the effect of ferrite bars which have been added in a following step. Using Ansys Maxwell FEM software several simulations were performed by varying the number of coils turns and the coil to coil distance. A fixed diameter of $D=40 \mathrm{~cm}$, was assumed. In Fig. 1 the coil geometric parameters are shown. The coil self-inductance $L$ decreases when increasing turn to turn distance $d$ while it increases when increasing the number of turns $N$. On the other hand, the parasitic resistance has an opposite behavior. It increases incrementing number of turns due to dc-resistance while it increases with the decreasing of distance between turns due to the proximity effect. Starting from the results of Fig.2, to guarantee a viable compromise between inductances and parasitic resistance, a pad composed of $N=15$ turn spaced with $d=4 \mathrm{~mm}$ corresponding to an inductance of $L=58 \mu \mathrm{H}$ was chosen.

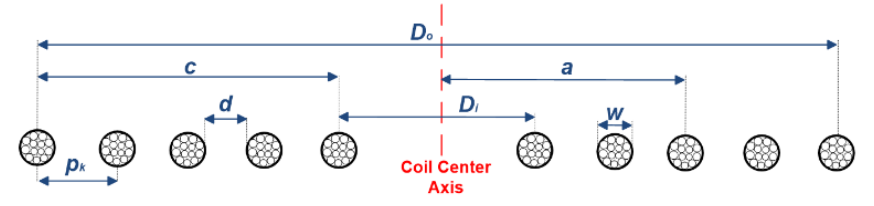

Fig. 1. Coil cross-section.

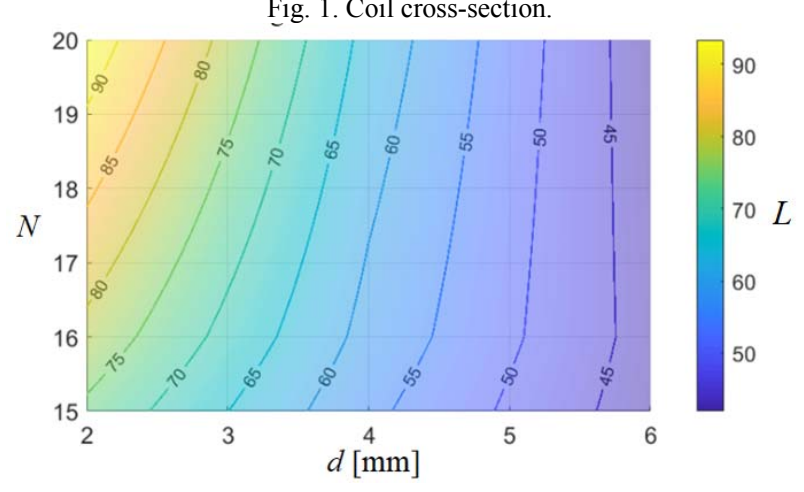

Fig.2. Coil inductance study result.

\section{Selection of Soft Ferrite Material}

In a circular coil, soft ferrite plates are usually used for two main reasons:

- Creating a low reluctance flux path and improve the coupling between coils.

- Reducing the magnetic field in the surrounding environment to guarantee the safety of the people according to the International Commission on NonIonizing Radiation Protection (ICNIRP) directives. For this purpose, an additional aluminum plate is used to improve structural robustness and provide magnetic shielding.

There are two types of ferrites: soft and hard ferrite. Soft ferrite has low coercivity, meaning that the magnetization of the material can easily reverse direction without dissipating much energy. This property makes them suitable to be used in high frequency applications. In contrast hard ferrite has high coercivity and high remanence after magnetization.

Being this application characterized by high frequency reverse material magnetization, soft ferrite is used. The most common soft ferrites are composed by a combination of Manganese and Zinc (MnZn) or Nickel and Zinc (NiZn). The NiZn ferrites have a very high resistivity and are usually used for frequencies over $1 \mathrm{MHz}$. The MnZn usually work at lower frequency, in general up to $3 \mathrm{MHz}$ [5]. Based on the project specifications shown in the previous section and on different magnetization curve of ferrites present in the market, a comparison aimed to identify the best ferrite for this application was carried out. Three different materials were compared as shown in Table I.

The $B-H$ hysteresis loop is a very useful guide for the comparison of different types of magnetic materials. One of the most important AC properties of ferrite is the core loss. The AC core loss is a function of the magnetic material, magnetic material thickness, magnetic flux density $B_{A C}$, frequency $f$, and operating temperature. Thus, the choice of the magnetic material is based upon achieving the best characteristic using the standard trade-off such as cost, size, and performance.

TABLE I. FERRITE MATERIALS

\begin{tabular}{|c|c|c|c|c|c|c|c|c|}
\hline Type & $\mu_{\mathrm{i}}$ & $\begin{array}{c}d_{g} \\
{\left[\mathrm{~kg} / \mathrm{m}^{3}\right]}\end{array}$ & $\begin{array}{c}\boldsymbol{T}_{\boldsymbol{c}} \\
{\left[{ }^{\circ} \mathbf{C}\right]}\end{array}$ & $\begin{array}{c}\mathrm{Br} \\
{[\mathrm{mT}]}\end{array}$ & $\begin{array}{c}H c \\
{[\mathbf{A} / \mathbf{m}]}\end{array}$ & $\begin{array}{c}B s \\
{[\mathrm{mT}]}\end{array}$ & $\begin{array}{c}\rho \\
{[\Omega \mathrm{m}]}\end{array}$ & $\tan (\delta) / \mu_{\mathrm{i}}$ \\
\hline $\begin{array}{c}\mathrm{L} 8 \mathrm{~F} \\
(\mathrm{NiZn})\end{array}$ & 1500 & 5100 & 130 & 130 & 30 & 330 & $10^{5}$ & $\begin{array}{c}60 \cdot 10^{-6} \\
\text { @ } 6 \mathrm{MHz}\end{array}$ \\
\hline $\begin{array}{l}\mathrm{HS12} \\
(\mathrm{MnZn})\end{array}$ & 12000 & 4900 & 130 & 80 & 6 & 430 & 0.5 & $\begin{array}{c}20 \cdot 10^{-6} \\
@ 0.1 \mathrm{MHz}\end{array}$ \\
\hline $\begin{array}{c}\text { M33 } \\
(\mathrm{MnZn})\end{array}$ & 2300 & 4700 & 200 & 55 & 9 & 390 & 5 & 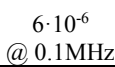 \\
\hline
\end{tabular}

The material L8F is NiZn, while the material HS12 and M33 are $\mathrm{MnZn}$. The L8F material exhibits higher ohmic resistivity $\rho$ but lower permeability $\mu_{i}$. It can work at higher frequency compared with the other ones, but the operating frequency is lower enough to exploit the advantages of MnZn materials. The choice is therefore between HS12 and M33. The HS12 has a very high permeability but a resistivity ten times lower than M33, leading to higher eddy current losses. For this reason, the M33 material is chosen because even though it has a lower permeability, it shows higher resistivity and lower density $d_{\mathrm{g}}$. For these reasons it is the most suitable for our purposes.

The power losses of a ferrite bar can be calculated with the following equation [9]

$$
P_{\text {core }}=C_{m} f^{\alpha} B_{\max }^{\beta}
$$

where $C_{m}, \alpha$, and $\beta$ are the constants which depend on the core properties. It indicates that the bar loss for ac magnetic field at any point in the ferrite is directly related with the frequency of the magnetic field and the peak flux density $B_{\max }$. 
The total bar loss is the volume integral of this loss in the transmitter and receiver bars, given as follows

$$
P_{\text {core }}^{\text {tot }}=C_{m} f^{\alpha} \int_{P A D} B_{\max }^{\beta} d V
$$

Being the frequency fixed to $f=85 \mathrm{kHz}$, once selected the ferrite type, the only parameter left for optimization is the flux density distribution in the bars. This means that it is possible to optimize the ferrite core losses working on the number of turns, the position and span of the coil as well as the permeability, geometry and most importantly the thickness of the core.

\section{Positioning and Optimization of Ferrite bars}

In [10], [11], the relative position between copper turns and ferrite bars that maximize the mutual inductance (minimizing the dispersed one) is shown. Starting from these results, a more in-depth analysis was carried out taking into account the ferrite nonlinear behavior when it is in saturation. Performing several FEM simulations, it was possible notice that the magnetic flux $B$ decreases along the ends of the bars, as shown in Fig. 3. These ferrite parts where the induction is very low are thus useless resulting in a waste of material. For this reason, the bars length was reduced letting them protrude only one centimeter with respect to the coil. An optimal length was $L=142.5 \mathrm{~mm}$.

\section{E. Thickness and width selection}

Once the bar length was selected, the attention was focused on the bar width and thickness. Using $\mathrm{N}=15$ turns, an outer diameter of $D_{0}=40 \mathrm{~cm}$ and $b=8$ bars with a length of $L=142.5$ $\mathrm{mm}$ an analysis on the self-inductance, the maximum magnetic flux densities and volume for different values of thickness and width was performed. In order to limit weight, size and cost of the pad, a thickness between 5 and $10 \mathrm{~mm}$ and a width between 25 and $40 \mathrm{~mm}$ was considered. The results shown in Fig. 4 show that if $25 \mathrm{~mm}$ width are chosen, the bars work close to saturation independently from the thickens. A good compromise between saturation and system weight is represented by a bar width of $35 \mathrm{~mm}$ and a thickness of $7.5 \mathrm{~mm}$. The magnetic flux density trough the bars is shown in Fig.5.

\section{F. Aluminum Shielding}

The Aluminum skin depth at a frequency $f=85 \mathrm{kHz}$, resistivity $\rho=2.75 \cdot 10^{-8} \Omega \mathrm{m}$, angular frequency $\omega=2 \pi f$ and permeability $\mu=1.25 \cdot 10^{-6} \mathrm{H} / \mathrm{m}$ is

$$
\delta=\sqrt{\frac{2 \rho}{\omega \mu}}
$$

The induced current density field into the aluminum plate does not vary significantly when increasing plate thickness, as long as thickness is greater than skin depth. For this reason, an aluminum plate of $1 \mathrm{~mm}$ thickness was used.

The system with the aluminum shielding was studied using the eddy current module. The simulation results are shown in Fig. 6. This is due to eddy currents of the aluminum shielding and implies a coupling reduction between pads. To avoid saturation, it has been necessary to increase ferrite bar thickness. Hence, ferrite bars with $9 \mathrm{~mm}$ thickness were used.
In fact, coil self-inductance without aluminum plate is $L_{l}$ $=105.57$ with a mutual inductance of $M=24.26 \mu \mathrm{H}$, while the self-inductance with aluminum plate is $L_{1}=84.26 \mu \mathrm{H}$ with a mutual inductance of $M=15.10 \mu \mathrm{H}$.

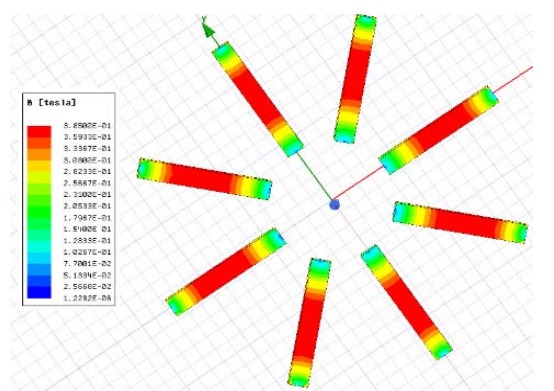

Fig.3 Magnetic field distribution across ferrite bars.

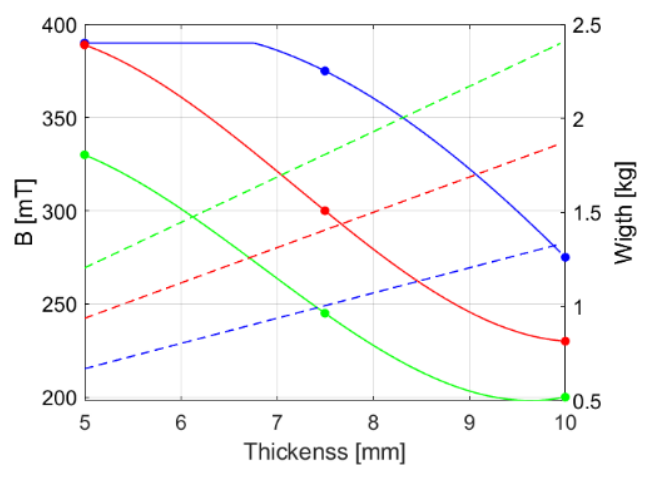

Fig. 4 Maximum Magnetic field and weight varying thickness. The blue trace is with $25 \mathrm{~mm}$ width, the red trace is with $35 \mathrm{~mm}$ width and the green trace is with $45 \mathrm{~mm}$ width

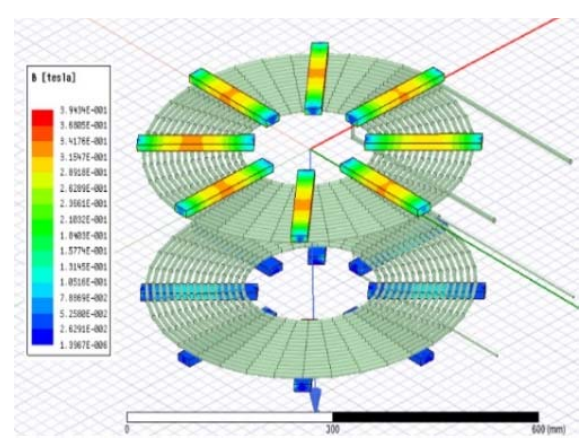

Fig.5. Flux density with $7.5 \mathrm{~mm}$ thickness and $35 \mathrm{~mm}$ width

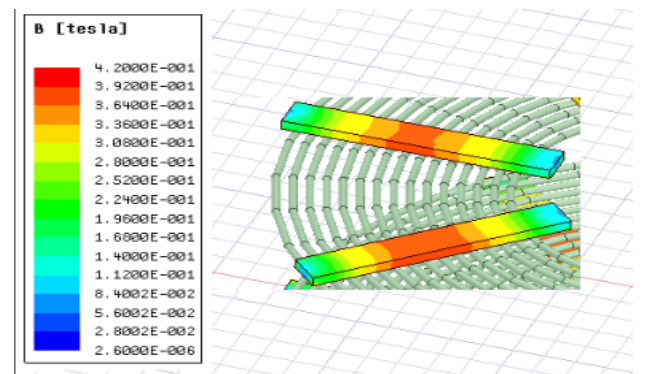

Fig.6. Ferrite bars saturation due to eddy currents introduced from the aluminum shielding. 


\section{HuMAN EXPOSURE OF ELECTROMAGNETIC FIELD (EMF)}

When a good compromise between performance, weight and costs was found, the electromagnetic field human exposure is analyzed to ensure the acceptable levels as specified in safety standard. There are two international groups that set standards/Guidelines for Human Exposure to Electromagnetic field (EMF): one is the International Committee on Electromagnetic Safety (ICES), and the other one is International Commission on Non-ionizing Radiation Protection (ICNIRP) [12].

For frequencies between $3 \mathrm{kHz}$ and $10 \mathrm{MHz}$, the reference levels for occupational exposure to time-varying magnetic fields is $100 \mu \mathrm{T}$ (rms), while reference level for public exposure to timevarying magnetic fields is $27 \mu \mathrm{T}$ (rms). Since in this case, the field of interest is wireless power transfer system for electric vehicle operated for private use it is reasonable to consider it under occupational exposure regulation.

The electromagnetic field exposure under the three case of Fig. 8 along the axis $l_{1}$ and $l_{2}$ is shown in Fig. 7(b) and (c).

In Fig.6, the red trace represents the measure of magnetic flux along the line $I_{2}$ of the coil without ferrite. The blue trace represents a measure of the magnetic flux with ferrite and without aluminum plate, while the yellow one represents the final case with ferrite and aluminum shield. The magnetic field lines in the three cases are shown in Fig. 6. It is interesting to see how the dispersed field was gradually reduced during the design steps. The yellow trace represents the pad without ferrite plates and shielding. The green trace represents the case with only ferrite plates, while the red trace represents the completed pad with ferrite and aluminum shield. The electromagnetic field exposure measured along the $l_{l}$ axis is shown in Fig. 6(a), while the exposure measured along the $l_{2}$ axis is shown in Fig. 6(b)

The axis $l_{2}$ is usually of greater importance regarding electromagnetic exposure because the driving position is usually placed above the coil. This is also the reason because the aluminum shielding is inserted to shield the electromagnetic field along $l_{2}$ axis. The effect of the aluminum shield is visible in Fig. 6(a). The EMF exposure is steadily under the occupational exposure limit only if aluminum shield is used. On the other hand, the EMF exposure is out of standard until $600 \mathrm{~mm}$ if the shielding it is not used.

The EMF exposure differences in the three cases along the $l_{l}$ axis are not marked. In both three cases the occupational exposure limits is reached after $570 \mathrm{~mm}$.

\section{IMPROVEMENTS AND OPTIMIZATION}

\section{G. Ohmic Losses Reduction}

A useful tool available in Ansys Maxwell is the ohmic losses calculator. The ohmic losses are calculated as

$$
P_{\text {ohmic }}=\frac{1}{2 \sigma} \iiint_{V} \operatorname{Re}(\vec{J} \cdot \vec{J}) d V
$$

The ohmic losses have three main contributions. Losses in the copper coil due to skin and proximity effect, losses in the ferrite bars due to the limited resistivity and the aluminum losses due to Foucault currents created from the alternate magnetic field.

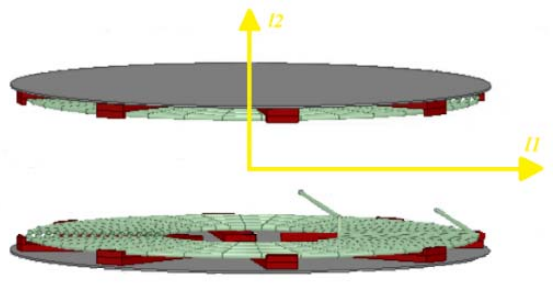

(a)

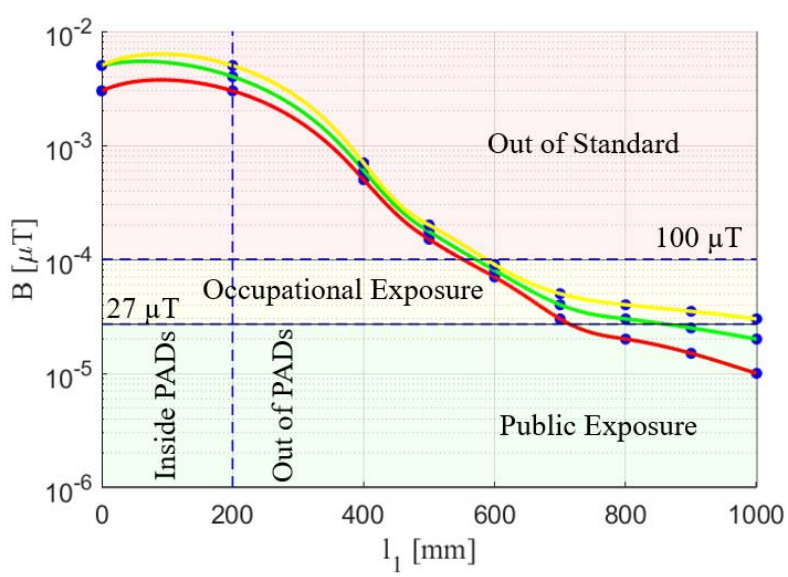

(b)

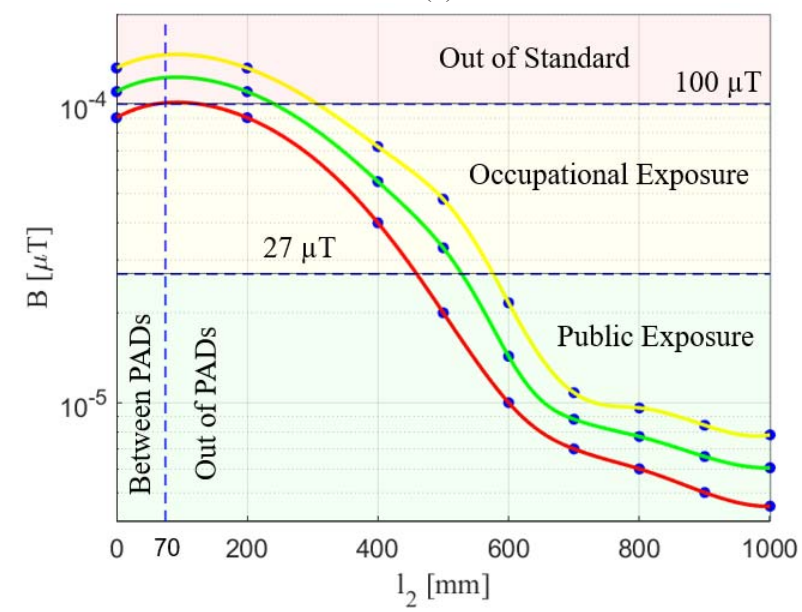

(c)

Fig.7. Comparison between coil with aluminum shield and ferrite (red trace), coil with ferrite (green trace) and only coil (yellow trace) EMF exposure along $l 1$ and $l_{2}$ axis. (a) Directions of the two axes. (b) Magnetic field along $l_{l}$. (c) Magnetic field along $l_{2}$.

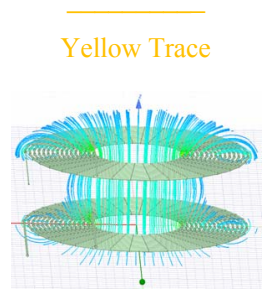

(a)

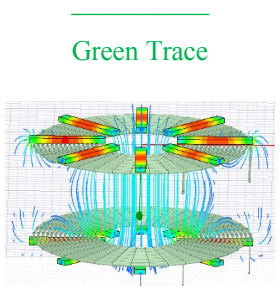

(b)

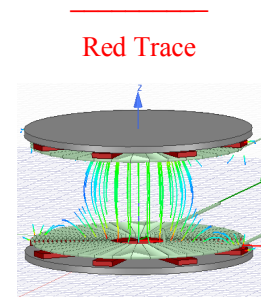

(c)
Fig. 8 (a) Dispersed field without ferrite bars and shields. (b) Dispersed field without shields. (c) Dispersed field with shield 

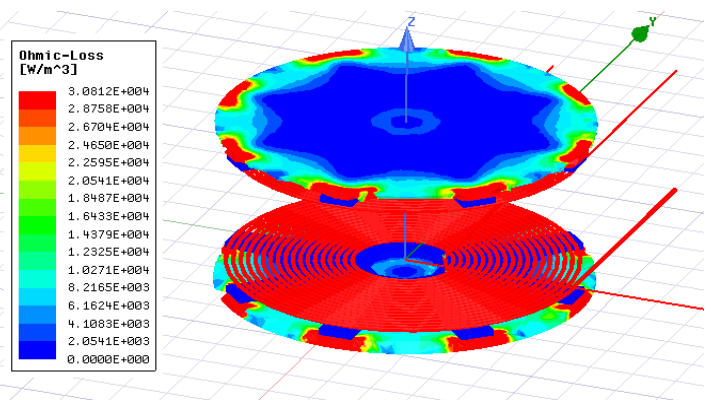

Fig. 9 PAD Ohmic losses distribution

From the simulation results shown in Fig.9 it is clear that the major ohmic losses are related to the copper coils and in the aluminum shield. The copper coil losses cannot be further reduced because the AWG strand, diameter and coil geometry were already optimized. By analyzing in detail the FEM results, a critical issue concerns the ferrite termination was highlighted. As shown in Fig.10, the magnetic field lines from the aluminum shield at the edge of the ferrite bars produce important ohmic losses which cause heating of the shield.

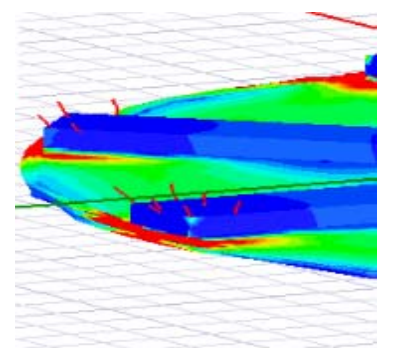

Fig. 10 Ohmic losses at bar termination

To hold this problem, a ferrite step at the termination was placed. This step is $35 \mathrm{~mm}$ long, of $7,5 \mathrm{~mm}$ high and has a width of $5 \mathrm{~mm}$.

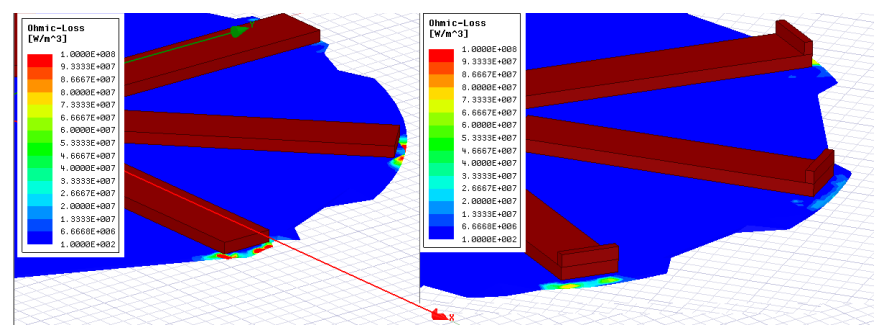

Fig.11. Improvements in ohmic losses

The addiction of the steps has improved the system efficiency reducing the losses due to the shield. The selfinductance with step is $L=88.64 \mu \mathrm{H}$ with a mutual inductance of $M=16.44 \mu \mathrm{H}$, while without step the self. Inductance is $L=$ $84.25 \mu \mathrm{H}$ with a mutual inductance of $M=15.01 \mu \mathrm{H}$.

More generally, these simulations show the importance of ferrite elements also to reduce shield induced losses and more generally losses due to induced currents on surrounding conductive elements. As consequence it should be concluded that a further refinement of ferrite flux guidance it's quite mandatory for real industrial applications, since reduced dispersed fluxes also produce a more robust performance and safety of the system respect to surrounding conductive or ferromagnetic components. This is a quite important specification also considering that this kind of materials are widely used on real vehicles.

\section{EXPERIMENTAL RESULTS}

To validate the FEM simulations, the system was reproducing in the laboratory. The experimental setup is shown in Fig. X. To measure the mutual inductance, the primary coil was supplied by a sinusoidal voltage and the induced voltage on the secondary side was measured with an oscilloscope. The value of the mutual inductance is

$$
M=\frac{L_{1} V_{2}}{V_{1}}
$$

The obtained results are shown and compared to the simulations in Fig. 12.

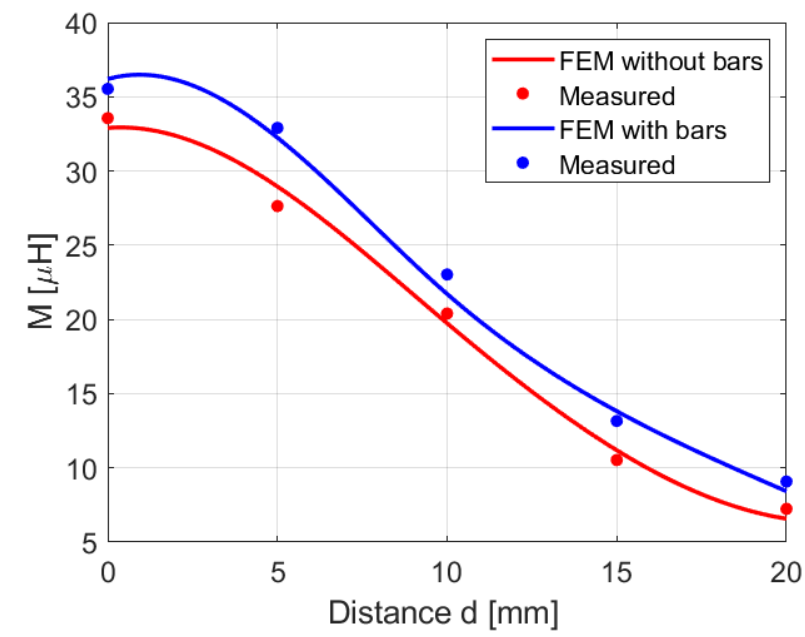

Fig. 12 Comparison between FEM simulations and experimental results.

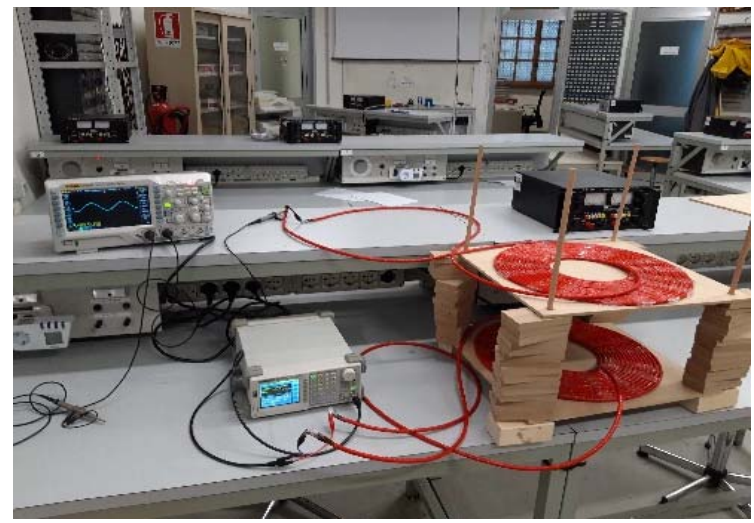

Fig. 13 Experimental Setup Measurements 


\section{CONCLUSIONS}

In this paper, the design and optimization of a wireless power transfer system is presented. The work has been carried out trying to obtain an optimal compromise among efficiency, low cost and low exposure of people to electromagnetic fields.

The activity has been performed by exploiting Ansys Maxwell finite element software. Power losses due to copper, ferrite bars and aluminum shielding are estimated. An innovative solution consisting on the addition of a ferrite step at the bar termination is presented. Experimental results validate the results obtained from the simulations.

\section{REFERENCES}

[1] A. Reatti et al., "Application of induction power recharge to garbage collection service," in RTSI 2017 - IEEE 3rd International Forum on Research and Technologies for Society and Industry, Conference Proceedings, 2017.

[2] L. Pugi, A. Reatti, and F. Corti, "Application of Wireless Power Transfer to Railway Parking Functionality: Preliminary Design Considerations with Series-Series and LCC Topologies," J. Adv. Transp., vol. 2018, pp. 1-14, 2018.

[3] B. Allotta, L. Pugi, A. Reatti, and F. Corti, "Wireless power recharge for underwater robotics," in Conference Proceedings 2017 17th IEEE International Conference on Environment and Electrical Engineering and 2017 1st IEEE Industrial and Commercial Power Systems Europe, EEEIC / I and CPS Europe 2017, 2017.

[4] C. Liu, C. Jiang, and C. Qiu, "Overview of coil designs for wireless charging of electric vehicle," in 2017 IEEE PELS Workshop on Emerging Technologies: Wireless Power Transfer, WoW 2017, 2017.

[5] Yo Sakaki and T. Matsuoka, "Hysteresis losses in Mn-Zn ferrite cores," IEEE Trans. Magn., vol. 22, no. 5, pp. 623-625, Sep. 1986.

[6] P. Bernardi, R. Cicchetti, G. Pelosi, A. Reatti, S. Selleri, and M. Tatini, "AN EQUIVALENT CIRCUIT FOR EMI PREDICTION IN PRINTED CIRCUIT BOARDS FEATURING A STRAIGHT-TO-BENT MICROSTRIP LINE
COUPLING," Prog. Electromagn. Res. B, 2008.

[7] D. K. Saini, A. Ayachit, A. Reatti, and M. K. Kazimierczuk, "Analysis and Design of Choke Inductors for Switched-Mode Power Inverters," IEEE Trans. Ind. Electron., vol. 65, no. 3, pp. 2234-2244, 2018.

[8] A. Ayachit, A. Reatti, and M. K. Kazimierczuk, "Magnetising inductance of multiple-output flyback dc-dc convertor for discontinuous-conduction mode," IET Power Electron., vol. 10, no. 4, pp. 451-461, 2016.

[9] M. Mohammad and S. Choi, "Optimization of ferrite core to reduce the core loss in double-D pad of wireless charging system for electric vehicles," in Conference Proceedings - IEEE Applied Power Electronics Conference and Exposition - APEC, 2018, vol. 2018-March, pp. 1350-1356.

[10] F. Castelli-Dezza, M. Mauri, A. Dolara, S. Leva, and M. Longo, "Power pad design and optimization for contactless electric vehicle battery charging system," in Conference Proceedings 2017 17th IEEE International Conference on Environment and Electrical Engineering and 2017 1st IEEE Industrial and Commercial Power Systems Europe, EEEIC / I and CPS Europe 2017, 2017.

[11] A. Dolara, S. Leva, M. Longo, F. Castelli-Dezza, and M. Mauri, "Coil design and magnetic shielding of a resonant wireless power transfer system for electric vehicle battery charging," in 2017 6th International Conference on Renewable Energy Research and Applications, ICRERA 2017, 2017, vol. 2017Janua, pp. 200-205.

[12] H. Jiang, P. Brazis, M. Tabaddor, and J. Bablo, "Safety considerations of wireless charger for electric vehicles - A review paper," in 2012 IEEE Symposium on Product Compliance Engineering, ISPCE 2012 - Proceedings, 2012, pp. 51-56. 